\title{
Testing the Gauss linear assumption for on-line predictions
}

\author{
Valentina Fedorova · Ilia Nouretdinov • \\ Alex Gammerman
}

Received: 30 November 2011 / Accepted: 17 June 2012 / Published online: 12 July 2012

(c) Springer-Verlag 2012

\begin{abstract}
The article studies prediction under some background assumptions and tests the assumptions. We use a conformal predictors framework and obtain predictions as regions. This is done by an on-line compression model that satisfies our assumptions. If the assumptions are appropriate for data, the prediction regions are automatically valid. The validity means that for successive predictions an error rate does not exceed a prefixed significance level (up to statistical fluctuation). It is important to test our assumptions to maintain the validity property; or otherwise the predictions can be misleading. We perform testing by calculating martingales. The martingales here are functions of $p$-values that are the output of the on-line compression model. Large values of the martingales reflect departures from the assumptions. This article extends our previous work on martingale testing and focusses on the Gauss linear assumption. The article presents experiments for the predictions and testing. We use four synthetic datasets as well as a benchmark Abalone dataset that is assumed to agree with the Gauss linear assumption.
\end{abstract}

Keywords Testing assumptions · Martingales · Gauss linear assumption · Conformal predictors

\section{Introduction}

This section briefly reviews results of the underlying theory and introduces necessary terminology.

V. Fedorova $(\varangle) \cdot$ I. Nouretdinov $\cdot$ A. Gammerman

Computer Learning Research Centre, Royal Holloway,

University of London, Egham, Surrey TW20 0EX, UK

e-mail: valentina@cs.rhul.ac.uk

I. Nouretdinov

e-mail: ilia@cs.rhul.ac.uk

A. Gammerman

e-mail: alex@cs.rhul.ac.uk

\subsection{Prediction with confidence}

Consider a classical problem of machine learning: having a training set of examples $\left\{\left(\mathbf{x}_{1}, y_{1}\right), \ldots,\left(\mathbf{x}_{n}, y_{n}\right)\right\}$, where $\mathbf{x}_{i}$ is called an object and $y_{i}$ is called its label, the goal is to predict a label for a new object $\mathbf{x}$. This article focuses on the regression problem where labels are real numbers.

Many algorithms (such as ridge regression, support vector machine, kernel methods and others) have been developed to deal with the regression problem. Their usual output is a bare prediction meaning that they do not provide any measure of confidence in it. Although Bayesian methods provide such guarantees, they use prior information. If the prior is incorrect then the predictions are misleading [3]. In this work we use the conformal predictors framework $[3,9,10]$ for two main reasons: firstly under some background assumptions it allows us to make predictions and supply corresponding confidence measures; secondly we employ the predictors to generate $p$-values that we use for testing the assumptions.

Conformal predictors, in general, output a prediction region. The region has to hold two important properties:

- validity states that the probability that the true label is outside the region does not exceed a significance level chosen beforehand;

- efficiency allows us to compare the usefulness of the prediction regions: the narrower a region is, the more efficient the prediction.

So, our main aim is to develop algorithms that produce valid prediction regions. In the on-line mode examples arrive one after another and predictions are obtained from the accumulated data. We observe a new object and use previous examples to output a prediction region. Then we observe a true value of its label and check our prediction. Practically, the 
validity of the predictions means that for a significance level $\varepsilon$ the ratio $\frac{\text { number of errors }}{\text { number of examples }}$ is about $\varepsilon$ or less.

\subsection{Testing}

To prove that our algorithms produce valid prediction regions we impose some assumptions on data. Therefore to guarantee that the validity of the predictions would not be violated it is important to test the assumptions. Usually the data are high-dimensional and traditional approaches to testing are inappropriate (see [7], pp. 6-7). In this article we describe some general testing ideas expressed in $[1,10]$ and apply them to the Gauss linear assumption.

The main tool of testing is a martingale. To calculate the martingale we first employ a conformal predictor which outputs a sequence of $p$-values for given data. Then we calculate a martingale as a function of the sequence. If a martingale grows to a large value the assumptions can be rejected with high probability.

It has been shown [9] that the conformal predictions are valid if data satisfy the exchangeability assumption. The martingale approach was introduced for testing the assumption. Actually we can test a slightly stronger assumption that the data is independent and identically distributed (i.i.d.), which implies the exchangeability assumption. By de Finetti's theorem (see [5], p. 28) any exchangeable distribution is a mixture of distributions under which data are i.i.d. So both of the assumptions have the same meaning for testing.

In this work we consider another assumption on data and use an on-line compression model (OCM) to obtain prediction regions and generate $p$-values. The OCM can be used with different assumptions, but this work deals with the Gauss linear assumption.

The rest of the article is organised as follows. There are two main parts: prediction under assumptions and testing the assumptions. In the first part, Sect. 2, we impose some assumptions on data and study predictions. We define an OCM and then consider OCM predictions for the Gauss linear assumption. To conclude we present experiments on predictions with synthetic datasets and discuss their properties. The second part of the article, Sect. 3, is devoted to testing the assumptions. We start by describing calculating of $p$-values by the OCM. Afterwards we define martingales and explain their use for testing. Then we describe calculation of the martingales that we use. Finally we report experiments on testing both synthetic and benchmark data. And in Sect. 4 we summarise the work.

\section{Prediction with OCM}

Data always can be considered following certain assumptions, for example, being i.i.d. or normally distributed or uni- formly distributed. To produce valid predictions we use an OCM which satisfies desired assumptions. We start with the general definition of an OCM and then consider the case of the Gauss linear assumption.

\subsection{On-line compression model (OCM)}

The theory of OCM is described in [9]. The OCM is constructed to extract information from data which is important according to our assumptions. It is called a summary of the data. The summary is analogous to the notion of sufficient statistics.

Formally an OCM consists of four elements:

1. $Z$ is an example space, that is where our examples come from;

2. $S$ is a summary space, its elements are called summaries;

3. $F_{n}: S \times Z \rightarrow S, n=1,2, \ldots$, that are called forward functions, these are rules to update a summary after observing a new example;

4. $B_{n}, n=1,2, \ldots$, that are called Backward kernels, these allow us to perform a step back and define a joint probability distribution of a previous summary and an example, conditional on a current summary.

In this work we use the OCM for the Gauss linear assumption. Next we reiterate the definition of the Gauss linear model and describe the corresponding OCM.

\subsection{Gauss linear model}

Consider examples of data as pairs $\left(\mathbf{x}_{n}, y_{n}\right)$, where $\mathbf{x}_{n}$ is a vector of $K$ attributes $\left(x_{n, 1}, \ldots, x_{n, K}\right)$ and $y_{n}$ is a number. Denote $\mathbf{z}_{n}=\left(1, \mathbf{x}_{n}\right)$, then the linear regression model has the form

$y_{n}=\boldsymbol{\gamma} \cdot \mathbf{z}_{n}+\xi_{n}$,

where $\boldsymbol{\gamma}=\left(\gamma_{1}, \ldots, \gamma_{K+1}\right)$ is a vector of $K+1$ unknown coefficients and $\xi_{n}, n=1,2, \ldots$ are random variables. For the Gauss linear model the random variables $\xi_{n}$ are assumed to be independent of each other and normally distributed with zero mean and a variance $\sigma^{2}>0$ (the same for all the variables).

\subsection{OCM for the Gauss linear assumption}

To specify the OCM for the Gauss linear assumption consider an example space as we have defined before in Sect. 2.2. 
Define a summary as

$$
\begin{aligned}
S_{n} & =S_{n}\left(\left(\mathbf{x}_{1}, y_{1}\right), \ldots,\left(\mathbf{x}_{n}, y_{n}\right)\right) \\
& =\left\{\mathbf{x}_{1}, \ldots, \mathbf{x}_{n}, \sum_{i=1}^{n} y_{i} \mathbf{x}_{i}, \sum_{i=1}^{n} y_{i}, \sum_{i=1}^{n} y_{i}^{2}\right\} .
\end{aligned}
$$

Update from $S_{n}$ to $S_{n+1}$ is straightforward: we augment $S_{n}$ with the object $\mathbf{x}_{n+1}$ and through term insert information about the example $\left(\mathbf{x}_{n+1}, y_{n+1}\right)$ into each of the sums

$$
\begin{aligned}
S_{n+1} & =S_{n+1}\left(S_{n},\left(\mathbf{x}_{n+1}, y_{n+1}\right)\right) \\
& =\left\{\mathbf{x}_{1}, \ldots, \mathbf{x}_{n}, \mathbf{x}_{n+1}, \sum_{i=1}^{n+1} y_{i} \mathbf{x}_{i}, \sum_{i=1}^{n+1} y_{i}, \sum_{i=1}^{n+1} y_{i}^{2}\right\} .
\end{aligned}
$$

The backward kernels define a joint distribution of $\left(S_{n-1},\left(\mathbf{x}_{n}, y_{n}\right)\right)$ for given $S_{n}$. According to the definition of the summary for the Gauss linear assumption $S_{n}$ contains all the observed objects $\mathbf{x}_{i}, i=1 \ldots, n$. Therefore for given $S_{n}$ the backward kernels $B_{n}$ actually describe a distribution of sequences $\left(\tilde{y}_{1}, \ldots \tilde{y}_{n}\right)$ that are consistent with the summary $S_{n}$. The sequences have to agree with $K+1$ linear equations (for each of $K+1$ coordinates of a vector $\mathbf{z}_{n}$ ) and the sum $\sum_{i=1}^{n} y_{i}^{2}$ is fixed. Consider a sphere which is an intersection of the $n$-dimensional sphere of radius $\sum_{i=1}^{n} y_{i}^{2}$ centred at the origin and the hyperplane $\left(c_{1}, \ldots, c_{n}\right)$ defined by:

$$
\begin{aligned}
\sum_{i=1}^{n} c_{i} & =\sum_{i=1}^{n} y_{i} \text { and } \sum_{i=1}^{n} c_{i} \cdot x_{i, p} \\
& =\sum_{i=1}^{n} y_{i} x_{i, p}, p=1, \ldots, K .
\end{aligned}
$$

Then the sequences $\left(\tilde{y}_{1}, \ldots \tilde{y}_{n}\right)$ distribute uniformly over the sphere of intersection. These sequences specify corresponding values for $S_{n-1}$ and $y_{n}$ that gives us the conditional distribution of $\left(S_{n-1},\left(\mathbf{x}_{n}, y_{n}\right)\right)$.

\subsection{OCM predictions}

\subsubsection{Prediction region for the Gauss linear assumption}

For each $l=1,2, \ldots$ set

$\mathbf{Z}_{l}=\left(\begin{array}{l}\mathbf{z}_{1}^{\prime} \\ \vdots \\ \mathbf{z}_{l}^{\prime}\end{array}\right) \quad$ and $\quad \mathbf{y}_{l}=\left(\begin{array}{l}y_{1} \\ \vdots \\ y_{l}\end{array}\right)$,

where $\mathbf{z}_{i}^{\prime}$ denotes the transposition of $\mathbf{z}_{i}$. Thus $\mathbf{Z}_{l}$ is a matrix size of $l \times(K+1), \mathbf{y}_{l}$ is a column vector of length $l$.

When $n-1>K+1$ and $\operatorname{Rank}\left(\mathbf{Z}_{n-1}\right)=K+1$ we can obtain a prediction region using Algorithm 1. First we estimate the regression coefficients from the observed examples by the least squares method. Then we use a well-known fact [4] that (2) has the $t$-distribution with $n-K-2$ degrees of

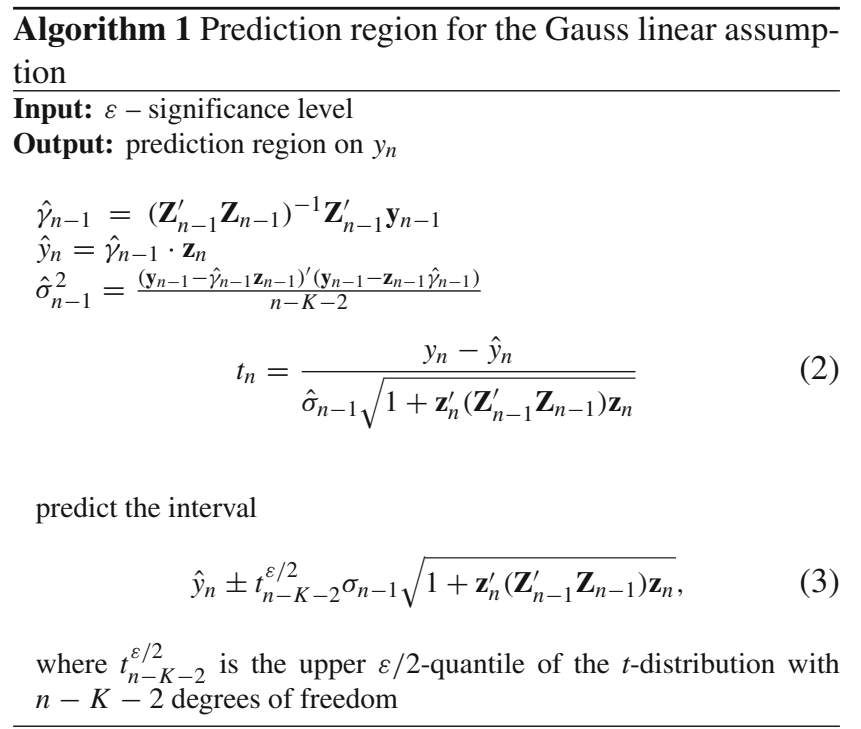

freedom and obtain the prediction region for a chosen significance level $\varepsilon$. From the definition of a quantile follows that the true label $y_{n}$ falls into the interval (3) with the probability $1-\varepsilon$. So Algorithm 1 outputs valid prediction regions.

\subsubsection{General way to make predictions by OCM}

The OCM can calculate prediction regions without help of $t$-distributed variable (2). First we define a sequence of realvalue measurable functions

$$
A_{n}=A_{n}\left(S_{n-1}\left(\left(\mathbf{x}_{1}, y_{1}\right), \ldots,\left(\mathbf{x}_{n-1}, y_{n-1}\right)\right),(\mathbf{x}, y)\right)
$$

that are called "nonconformity" measures. Based on the prediction algorithm each of the functions estimates strangeness of a pair $(\mathbf{x}, y)$ regarding a summary $S_{n-1}$. A natural nonconformity measure for the model (1) is

$A_{n}\left(S_{n-1},(\mathbf{x}, y)\right)=|y-\hat{y}|$,

where $\hat{y}$ is the least squares estimate of $y$. Then to obtain a prediction region for the significance level $\varepsilon$ we follow Algorithm 2. There we try all possible values $y^{*}$ as a label of the new object $\mathbf{x}$. Then we estimate a $p$-value for the label and form the prediction region using the labels that provide $p$-values higher than the chosen level.

Next we discuss a calculation of the probability (5) by Monte-Carlo simulation. Algorithm 3 summarises the method. For every iteration we generate a random vector instead of $\left(y_{1}, \ldots, y_{n-1}, y^{*}\right)$ regarding $S_{n}$. Consider the vector as coordinates of a point in the $n$-dimensional space. Then to estimate (5) we generate points that distribute uniformly over an $(n-K-1)$-dimensional sphere. We construct each point from an $n$-dimensional normally distributed point a (see step 1 of Algorithm 3). A summary $S_{n}$ defines a $(K+1)$ dimensional hyperplane and an $n$-dimensional sphere. First 


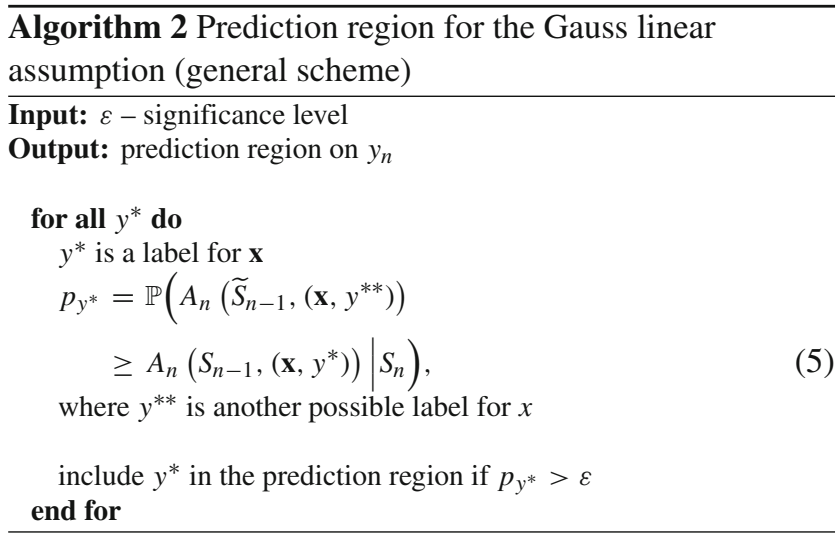

we project a onto the $(n-K-1)$-dimensional space where an intersection of the sphere and the hyperplane lies. Then we find the position and radius of the sphere of intersection: we calculate the distance between the hyperplane and the origin and then use the Pythagorean theorem. Finally to get the desired property for the point a we move it onto the sphere. Then we use the coordinates of the point as labels of our objects and calculate corresponding "nonconformity" score. To estimate the probability (5) we compare the"nonconformity" scores of the generated labels with the actual "nonconformity".

2.4.2.1 Computational cost To estimate $p_{y^{*}}$ by Algorithm 3 we perform Monte-Carlo simulation. We use $M=$ 100 samples to obtain acceptable precision of the $p$-value. Steps 2 and 4 can be computed once for each $y^{*}$. There we perform an orthonormalisation, that by the Gram-Schmidt process has $O(n)$ operations, and we solve the quadratic programming problem, that is usually a polynomial time $O\left(n^{p}\right)$ for some constant $p$. In the next evaluations we assume that $n \gg K$. Then for each iteration we perform $O\left(n^{2}\right)$ multiplications and additions in step 3, $O(n)$ multiplications and additions in step 5. Altogether computing $p$-values accurate to $\frac{1}{M}$ has the complexity $O(n)+O\left(n^{p}\right)+M \cdot(O(n)+$ $\left.O\left(n^{2}\right)\right)=O\left(n^{p}\right)+O\left(n^{2}\right) \geq O\left(n^{2}\right)$, where $n$ is the number of observed examples.

The outputs of Algorithms 1 and 2 coincide. In the first method the prediction regions are obtained without explicit calculation of $p$-values. So Algorithm 1 is faster, but it is based on the properties of the Gauss linear model. We have described Algorithm 2 to show the general process of obtaining predictions by an OCM. In a similar way we can make OCM predictions for other assumptions as well.

\subsection{Experiments}

In this section we demonstrate predictions on synthetic and randomly generated datasets. We evaluate validity and efficiency of the predictions when the data are generated

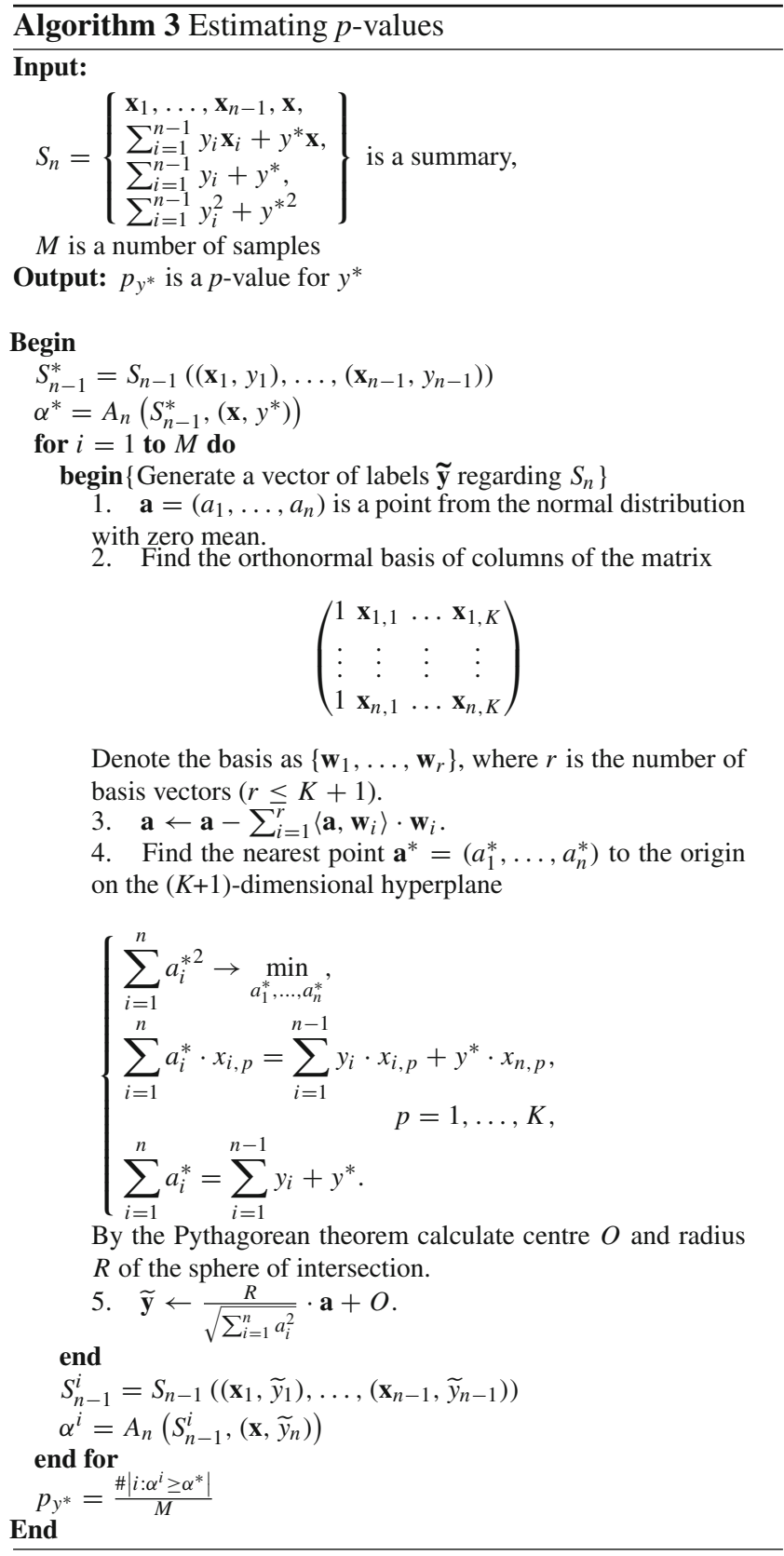

according to the Gauss linear model as well as when the assumption is violated.

\subsubsection{Synthetic datasets}

For the next experiments we use four different datasets. The datasets have $N=2,000$ examples. Each example is described by $K=100$ explanatory attributes and a label. The attributes have been generated independently from the normal distribution with zero mean and variance one. The label is a dependent attribute and has been calculated using 
(1) with the vector of coefficients

$\gamma=(100, \underbrace{10,-10 \ldots 10,-10}_{10}, \underbrace{1,-1 \ldots 1,-1}_{90})$, i.e.

$\gamma_{k}= \begin{cases}100, & k=1 ; \\ (-1)^{k+1} \cdot 10, & k=2, \ldots, 11 \\ (-1)^{k+1}, & k=12, \ldots, 101 .\end{cases}$

According to the settings the first ten attributes are more important than the rest. For the GaussData dataset the noise variables $\xi_{i}$ have been generated from the normal distribution with zero mean and variance one. For the LaplaceData, ExponentialData and UniformData datasets $\xi_{i}$ have been drawn respectively from the Laplace distribution with location parameter zero and scale parameter $\frac{1}{\sqrt{2}}$, the exponential distribution with rate parameter one and the uniform distribution on $[-\sqrt{3}, \sqrt{3}]$. The parameters of the distributions have been chosen to result in variance one and mean zero (except the exponentially distributed noise where mean is one).

Figure 1 illustrates the validity property of the predictions obtained by the OCM for the Gauss linear assumption. For the datasets the number of attributes is $K=100$ so we start to obtain on-line predictions by Algorithm 1 from an example with the index $K+3=103$ (see Sect. 2.4.1).

We set the significance level to $5 \%$ and calculate error rates in the on-line mode. The validity for the datasets and the significance level means that after 1897 successive on-line predictions (starting from the index 103) an overall number of errors does not exceed $1897 \times 0.05 \approx 95$. The dashed line (expected for level $5 \%$ ) in Fig. 1 shows this allowed number of errors for the on-line predictions. The graphs support theoretical expectations: when the assumption is satisfied for data (the GaussData dataset) the error rate (the solid line) is about the significance level of $5 \%$ (up to statistical fluctuation).

In Fig. 1 we can see different error rates when the underlying assumption is violated: for the LaplaceData and ExponentialData datasets (the line with dots and the line with squares) the error rates are higher than it is expected, whereas for the UniformData dataset (the line with triangles) the number of errors is lower than we expect.

Next Fig. 2 illustrates the efficiency of the predictions for the GaussData dataset. Theoretically, if a distribution is approximately Gaussian then about $95 \%$ of values fall within two standard deviations around mean. In our settings (the standard deviation for the data is 1) it would give a region of width 4 and the OCM generates regions of width 4.2 as soon as a sufficient number of examples has been observed.

In Fig. 1, prediction quality (the number of errors) for the UniformData dataset seems better than for the GaussData dataset. This is because of some properties of the uniformly distributed noise. The uniform distribution is symmetric and platykurtic so there is no significant departure from the Gauss

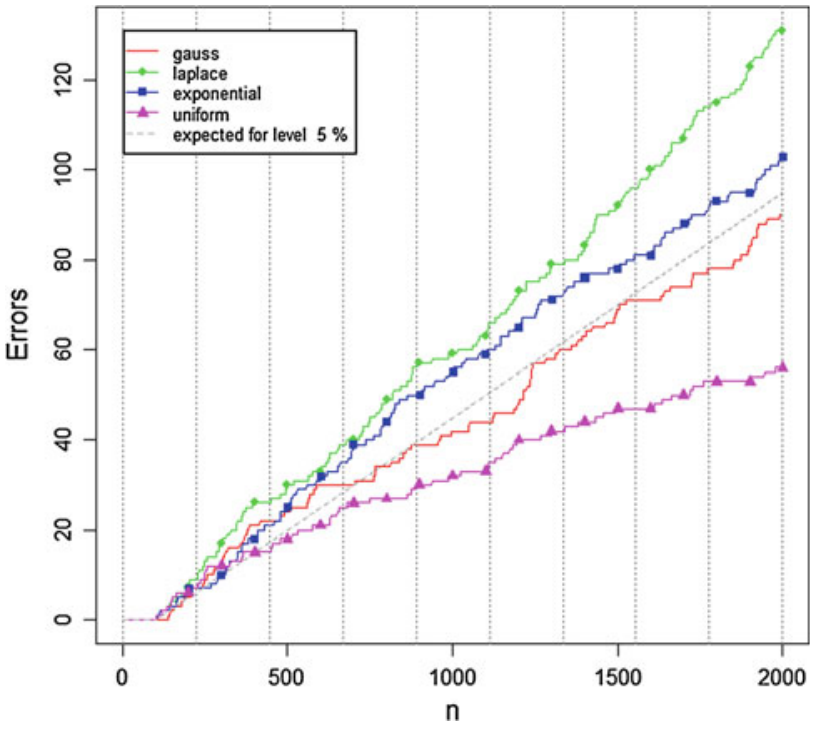

Fig. 1 Error rates are plotted against $n$ for four synthetic datasets (the significance level is $5 \%$ )

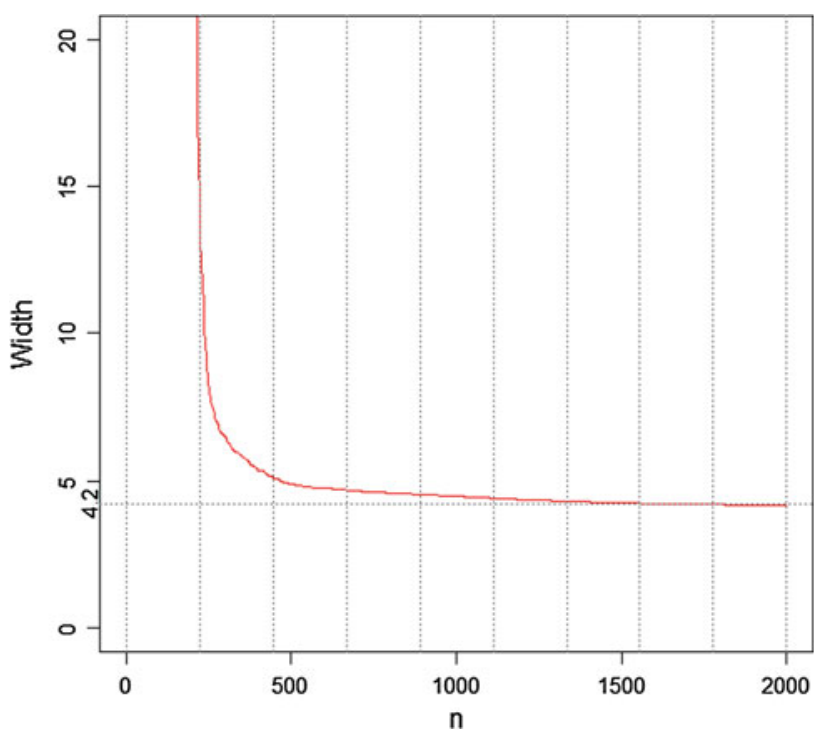

Fig. 2 Median widths of prediction regions are plotted against $n$ for GaussData (the significance level is $5 \%$ )

distribution. Also the uniform distribution has zero tails and therefore puts stronger bounds on possible values than the Gauss distribution does. But of course, this particular result for UniformData might not be relevant in general, there is no guarantee of the validity for other datasets with a uniformly distributed noise.

The next section of the article is devoted to the problem of testing underlying assumptions. There we describe an on-line testing approach and show that it detects even small deviations from the Gauss linear assumption. As Fig.1 shows the results of testing are important to be sure that the validity of OCM predictions would not be violated. 


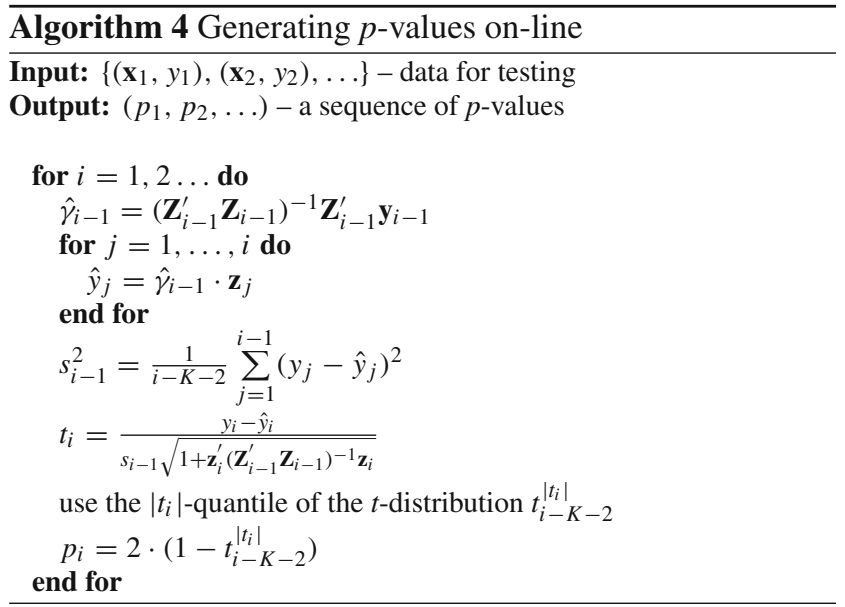

\section{On-line testing}

In this section we present on-line testing: the procedure provides a number which reflects a degree of deviation from our assumptions after observing every example of data. Following [10] we calculate a martingale and when the martingale value is large the assumptions are rejected. Our martingales are functions of $p$-values. For testing the Gauss linear assumption the p-values are output by the OCM. We start by describing calculation of the $p$-values and then give some intuition behind martingales in general and the plug-in martingale particularly.

\subsection{Generating $p$-values by OCM}

Consider a sequence of examples $\left\{\left(\mathbf{x}_{1}, y_{1}\right),\left(\mathbf{x}_{2}, y_{2}\right) \ldots\right\}$. To translate it into a sequence of $p$-values the OCM outputs the $p$-values that correspond to the true labels of the examples. The calculations are similar to obtaining a prediction region in Sect. 2. Again, there are two possible ways to calculate a $p$-value: either we use (5) in Algorithm 2 or we construct the $t$-distributed variable (2) in Algorithm 1. Next we describe the second approach that allows to calculate a $p$-value without expensive computations of Monte-Carlo simulation.

Consider examples $\left\{\left(\mathbf{x}_{1}, y_{1}\right), \ldots,\left(\mathbf{x}_{i-1}, y_{i-1}\right)\right\}$ and an example $\left(\mathbf{x}_{i}, y_{i}\right)$. To find a $p$-value $p_{i}$ corresponding to $y_{i}$ we calculate the value $t_{i}$ of the random variable (2). Denote $t_{i-K-2}^{\left|t_{i}\right|}$ for the $\left|t_{i}\right|$-th quantile of the $t$-distribution with $i-K-2$ degrees of freedom. Then the $p$-value is

$p_{i}=2\left(1-t_{i-K-1}^{\left|t_{i}\right|}\right)$.

To be more precise we use the same notation as in Algorithm 1 and Algorithm 4 summarises the calculation of the $p$-values.

In Algorithm 4 we use matrix operations to compute a p-value so its complexity is polynomial in the number of examples $n$.
In the theory of conformal predictors the next result has been proven (see [9], Theorem 8.1).

Theorem 1 If data agree with assumptions of the OCM then p-values output by Algorithm 4 are independent and distributed uniformly in $[0,1]$.

This property allows us to test the data on satisfying the OCM assumptions by constructing martingales that are functions of the $p$-values.

\subsection{Martingale}

Our goal in on-line testing is to get a number that reflects the strength of our belief in the assumptions. The number is provided by martingales. Next is the general intuition about the martingales.

Let us imagine a tossing coin game. A gambler places bets on head or tail: he does not bet more than the current capital and never risks bankruptcy. The gambler would like to test the coin on fairness. One way to do this is to place bets according to some strategy against the fairness of the coin. Suppose he decides to bet on the head. If the chosen strategy leads to a large growth of his initial capital then either the coin is biased or a rare event for the fair coin has happened. In the game, the strategy of the gambler can be thought as a martingale.

To define martingales formally let $z_{1}, z_{2}, \ldots$ be drawn from a probability distribution $\mathbf{P}$. Then a sequence of nonnegative random variables $M_{0}, M_{1}, \ldots$ is a martingale if each $M_{n}$ is a measurable function of $z_{1}, \ldots, z_{n}$ and the conditional expectation of $M_{n+1}$ given $M_{0}, \ldots, M_{n}$ is equal to $M_{n}$

$M_{n}=\mathbb{E}\left(M_{n+1} \mid M_{1}, \ldots, M_{n}\right)$,

where $\mathbb{E}$ refers to the expectation of $M_{n+1}$ in the probability space $\mathbf{P}$ that generates $z_{1}, z_{2}, \ldots$

According to Ville's inequality (see [8], p. 100) it is unlikely for any $M_{n}$ to have a large value

$\mathbf{P}\left\{\exists n: M_{n} \geq C\right\} \leq 1 / C, \forall C>0$.

Applying the fact we can test the assumption that data are drawn from the distribution $\mathbf{P}$. If the final value of the martingale is large then the assumption for the data can be rejected with a corresponding probability.

\subsection{Plug-in martingales}

The martingales that we use are functions of $p$-values and called the plug-in martingales. The martingales were introduced in [1]. Next we give some intuition behind them and describe their calculation.

As we have explained a martingale can be thought of as a betting strategy. The plug-in martingale bets according to the probability density function of observed $p$-values. If the 
distribution has any peak then it is used to bet when a new $p$-value arrives. Such martingale (under reasonable and mild assumptions) asymptotically grows better than other martingales that have been introduced. The martingale properties have been proven in [1] and next we outline calculation of the martingale.

The plug-in martingale has a form

$M_{n}=\prod_{i=1}^{n} \hat{\rho}_{i}\left(p_{i}\right)$,

where $\left\{p_{1}, \ldots, p_{i}\right\}$ are $p$-values output by the OCM (Algorithm 4) and $\hat{\rho}_{i}$ is an estimate of the probability density function calculated from $\left\{p_{1}, \ldots, p_{i-1}\right\}$. For the estimate we need $\int_{0}^{1} \hat{\rho}_{i}(p) \mathrm{d} p=1$ then (6) is a martingale. The estimate is recalculated for every iteration of the testing and the martingale is updated using the new estimate.

\subsubsection{Density estimation}

For the experiments presented in this article we have used statistical environment and language $\mathrm{R}$. The density estimate was calculated by the dens i ty function in the Stat s package. The function computes the kernel density estimate. For the results presented in the article the parameters used are the Gaussian kernel and Silverman's "rule of thumb" for bandwidth selection.

Next we show performance of the martingale with several datasets.

\subsection{Experiments}

In this subsection we present testing of datasets. First we use synthetic datasets to show that the procedure leads to reasonable and expected results. Second we present testing of benchmark data and obtain conformal predictions for the data as well.

\subsubsection{Synthetic dataset}

In Sect. 2.5 the four datasets (GaussData, LaplaceData, ExponentialData and UniformData) have been described. According to the way of generating the datasets only the GaussData dataset agrees with the Gauss linear assumption and for the rest of three datasets the assumption is violated. So we expect the martingales to keep small values for the GaussData dataset but grow significantly for the rest of the three datasets.

Figure 3 shows the martingale growth for the datasets. First we generate $p$-values in the on-line mode by Algorithm 4 . Then we calculate the martingale growth: the $p$ values are observed one after another and the martingale (6) is calculated. The vertical axis in Fig. 3 is for the logarithm

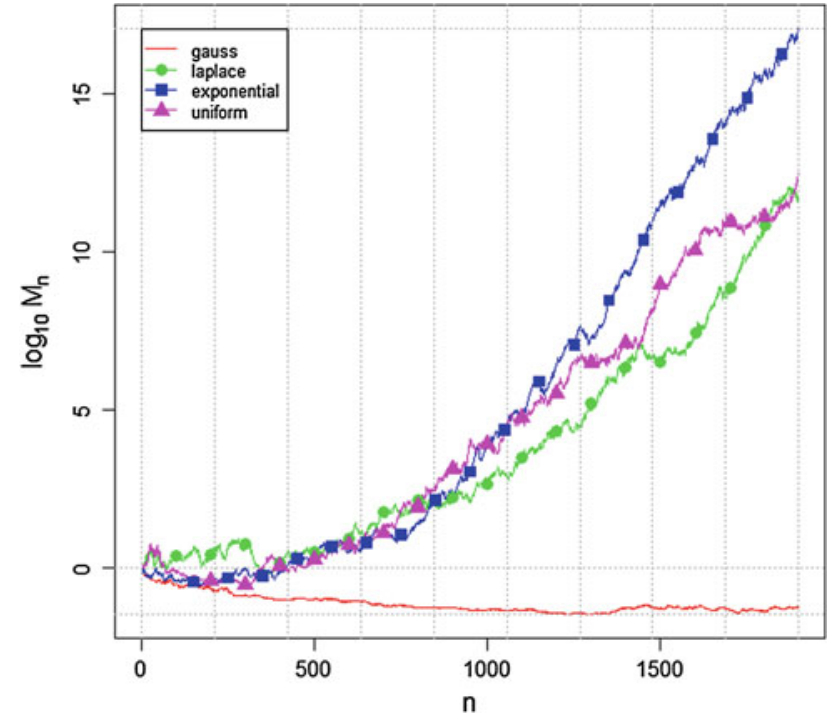

Fig. 3 Plug-in martingales show testing of the Gauss linear assumption for four synthetic datasets

of martingale and the horizontal one is for indices (number of observed examples so far). The solid line shows the martingale for the GaussData: the logarithm of martingale takes negative values that means the martingale values are about zero. For the rest of the three datasets (lines with different characters) the martingale final values are more than $10^{11}$. So the testing detects the departures from the Gauss linear assumption but does not reject the model for GaussData.

\subsubsection{Abalone dataset}

To demonstrate testing of a benchmark dataset we use Abalone data from the UCI repository [2]. The dataset addresses the problem of prediction the age of abalone from various physical measurements.

We consider this as a regression problem with $K=7$ numerical attributes and a numerical label; overall number of examples is 4,177 . Using the results from [6] we have decided to check two assumptions:

1. The age of abalone depends on the attributes according to the model (1) (described in Sect. 2.2) with the normally distributed noise.

2. The same as 1 but instead of the age we use the logarithm of abalone age.

Figure 4 shows the martingale growth for the assumptions. The final martingale value is $10^{36}$ for the assumption 1 and it is $10^{3.5}$ for the assumption 2. So the assumption 2 seems to be more realistic.

Figure 5 shows error rates for predictions based on the assumption 2 and for the significance levels of 5 and $1 \%$. 


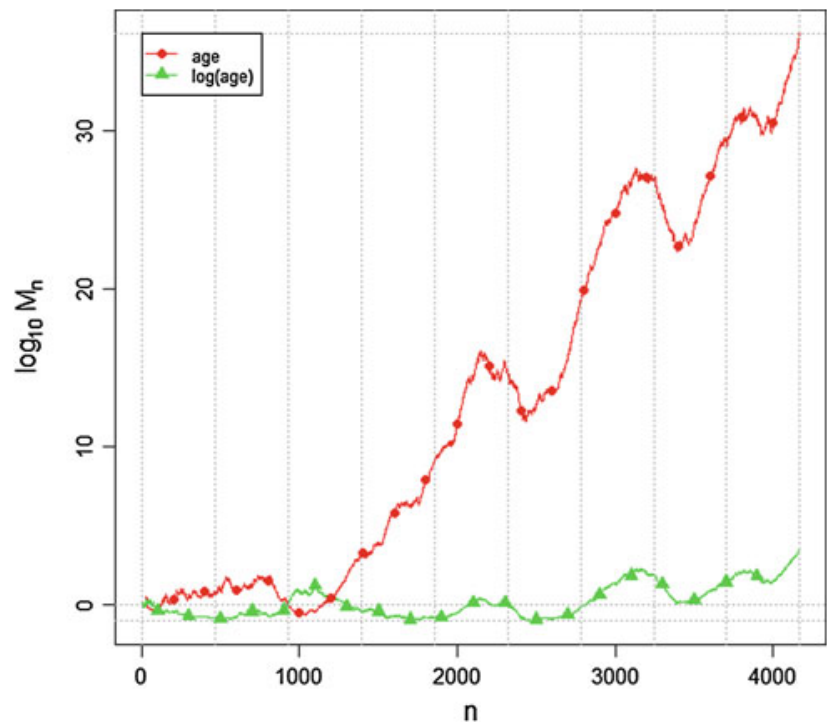

Fig. 4 Plug-in martingales show testing of the Gauss linear assumption for the abalone age or the logarithm of abalone age

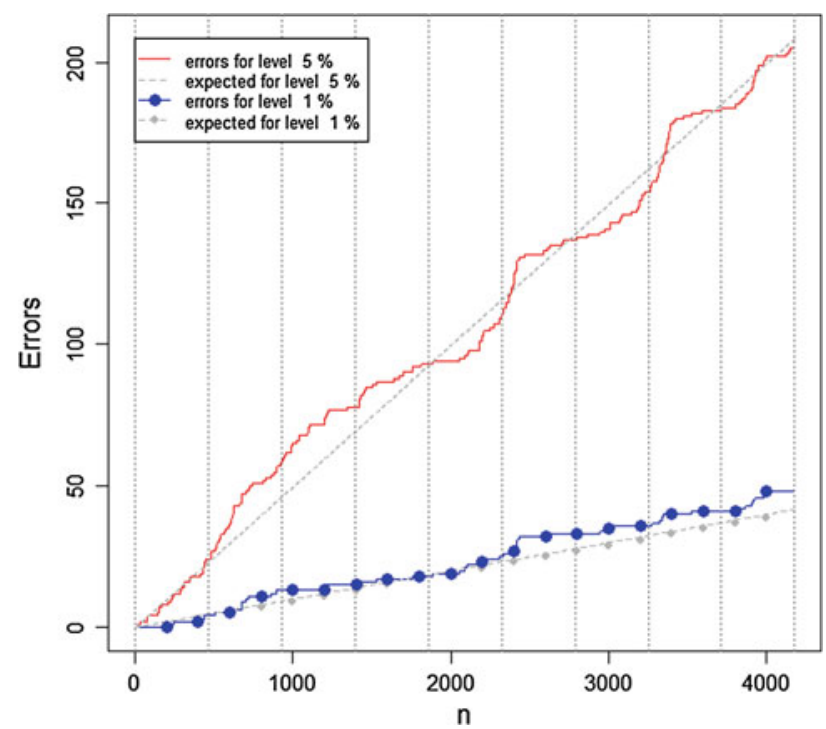

Fig. 5 Error rates are plotted against $n$ for predictions on the logarithm of abalone age (significance levels are 1 and $5 \%$ )

The number of errors for the predictions (the solid dark line and the dark line with dots) are close to the expected number of errors for the chosen significance levels (the solid grey line and the grey line with dots). In other words, the results are practically valid (in the sense mentioned in Sect. 1.1). We illustrate the efficiency of predictions by calculating the median width of prediction regions. Figure 6 shows the widths of prediction regions for the significance levels.

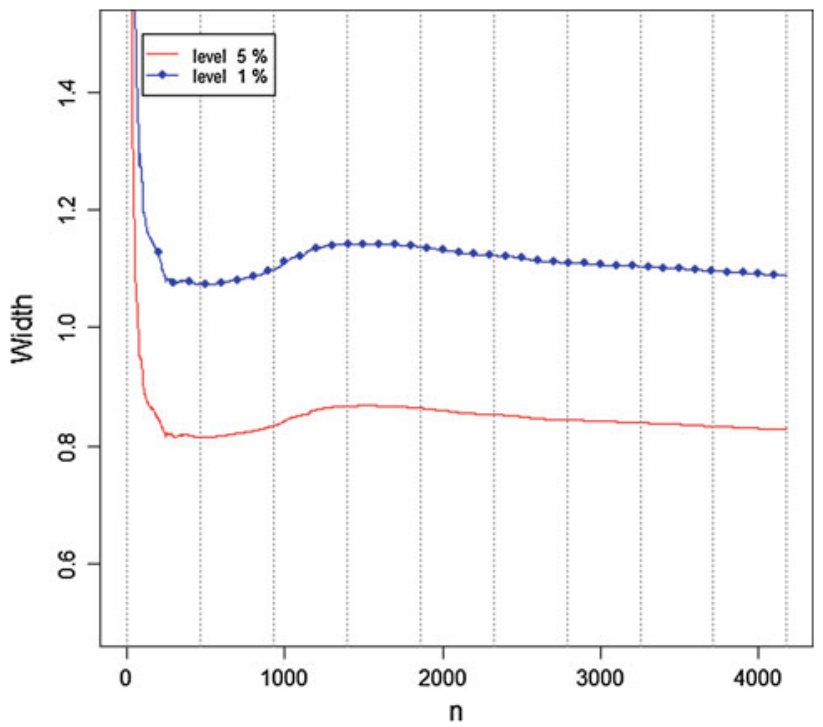

Fig. 6 Median widths of prediction regions for the logarithm of abalone age are plotted against $n$ (the significance levels are 1 and $5 \%$ )

\section{Conclusion}

In this article we have studied OCM predictions under the Gauss linear assumption and testing the assumption.

We have described the OCM under the assumption and have shown two ways to calculate prediction regions. Our experiments illustrate properties of the predictions for both cases when data agree with the accepted model as well as when the assumption is violated.

Then we have described on-line testing of the assumption. We have used the plug-in martingale and have shown its performance. The results support our theoretical expectations: even for small departures from the assumption the final martingale values are large (it is more than $10^{11}$ for the datasets of 2,000 examples) and the martingale value is close to zero when the assumption is satisfied.

To show the benefit of martingale testing let us compare Figs. 3, 1: the martingale extracts information about agreement of data with the Gauss linear assumption that can not be seen clearly from tracking numbers of errors in predictions.

We have presented testing the Gauss linear assumption on a benchmark Abalone dataset. Then predictions on the logarithm of abalone age have been obtained by the OCM. Again, the validity and efficiency for the predictions have been shown.

Though the article mainly discusses predictions and testing for the Gauss linear assumption this approach is wider in the sense that OCM predictions and martingale testing are adaptable to other assumptions analogously.

Acknowledgments We would like to thank Volodya Vovk for insightful discussions of the research problem. We are indebted to Royal Holloway, University of London, for continued support and funding. 
This work has also been supported by the EraSysBio+ grant SHIPREC from the European Union, BBSRC and BMBF and by the VLA grant on machine learning algorithms. We thank reviewers for their advice on improving the article.

\section{References}

1. Fedorova, V., Nouretdinov, I., Gammerman, A., Vovk, V.: Plug-in martingales for testing exchangeability on-line., In: Proceedings of the 29th International Conference on Machine Learning (ICML 2012), Edinburgh, Scotland, UK (2012)

2. Frank, A., Asuncion, A.: UCI repository, 2010. Accessed 19 May (2012)

3. Gammerman, A., Vovk, V.: Hedging predictions in machine learning. The Computer Journal. 50(2), 151-163 (2007)
4. Gosset, W.S. (Student): The probable error of a mean. Biometrika, 6, 1-25 (1908)

5. Schervish, M.J.: Theory of statistics. Springer, New York (1995)

6. Snelson, E., Rasmussen, C.E., Ghahramani, Z.: Warped Gaussian processes. In: Advances in Neural Information Processing Systems 16 (NIPS), (2004)

7. Vapnik, V.N.: Statistical learning theory. John Wiley \& Sons, Inc., New York (1998)

8. Ville, J.: Etude critique de la notion de collectif. GauthierVillars, Paris (1939)

9. Vovk, V., Gammerman, A., Shafer, G.: Algorithmic learning in a random world. Springer, New York (2005)

10. Vovk, V., Nouretdinov, I., Gammerman, A.: Testing exchangeability on-line. In: Proceedings of the 20th International Conference on Machine Learning (ICBM 2003), pp. 768-775, Washington, DC, (2003) 